\title{
Escape and avoidance responding in the toad
}

\section{F. T. CRAWFORD AND JAMES W. LANGDON}

FLORIDA STATE UNIVERSITY

Southern toads, Bufo terrestris terrestris, were given 20 daily trials in a one-way avoidance box for five days. The frequency of no responses decreased, avoidance responses increased, and escape responses remained essentially the same. There was an increase in the number of avoidance responses in each daily session of trials, followed by a lower initial level of avoidance responding on the following day. An improvement in performance as measured by response latency was also observed. The pattern of response latencies was consistent with that of the avoidance responses in that there was a decline in response latency over daily trials with higher initial levels on the early trials of the following day.

Conditioning in amphibians has been recently investigated by MoGill (1960). He observed escape latencies made by leopard frogs, Rana pipiens, when presented with electric shock. The frogs were tested in a shuttle box and demonstrated an increase in escape latency with continued training. A higher shock level reinstituted escape responding but this again was followed by a reduction in responsiveness. Subsequent increases in shock levels produced the same effects. This led McGill to conclude that the frogs demonstrated maladaptive learning in that all the experimental animals eventually died. In the present study a procedure is described which results in a consistent escape, and, an increasing degree of avoidance, responding.

\section{Method}

Ss of the study were 16 Southern toads, Bufo terrestris terrestris. They were maintained in an air conditioned room having a mean temperature of $25.5^{\circ} \mathrm{C}$ at $50 \%$ humidity. The room was illuminated $24 \mathrm{hr}$. a day. The toads were housed in terrariums containing moistened wood chips. Water was available ad lib and their diet consisted of grasshoppers, crickets, and mealworms. They were divided into two equal groups, one experimental and one control. Identification of individual toads was made possible by differentially clipping the toes.

Recent studies (e.g., Theios \& Dunaway, 1964) have confirmed the generally recognized superiority of the one-way avoidance chamber over that of the shuttle box. Testing was done, therefore, in a one-way escape box. The test chamber was a rectangular plastic box open on the bottom, and having a length, height, and width of 22.3, 5.3 , and $6.7 \mathrm{~cm}$, respectively. The end of one-half of the apparatus was made semi-transparent by lightly spraying it with flat white paint. The other one-half of the apparatus was lightly sprayed with flat black paint. The two portions of the apparatus were separated by a black guillotine door. Over the end, and on top, of the white end of the apparatus was a
$4.5 \times 4.5 \times 5.0 \mathrm{~cm}$ lamp housing having a square base and containing a $28 \mathrm{v}$ DC $1.56 \mathrm{w}$ bulb. The apparatus rested upon a grid made of strips of galvanized sheet metal. Alternate strips in the grid were wired together and the entire grid was held by a bakelite frame. The grid was wired so that when the test chamber was positioned over the grid, shock could be applied to the area under the black portion of the apparatus. The shock source, a Grass SD 5 stimulator, was set to deliver $40 \mathrm{v} \mathrm{DC}, 3$ pulses/sec. with $10 \mathrm{msec}$. duration. There was a $47 \mathrm{~K} \mathrm{ohm} \mathrm{series} \mathrm{resistance} \mathrm{in} \mathrm{the} \mathrm{shock} \mathrm{circuit.}$

Prior to each day's series of trials $S$ was rinsed off to remove wood chips, and placed on paper towels to remove excess water. $S$ was then placed in the black portion of the apparatus with the stimulus lamp over the white area turned on. On each trial the $S$ had to be stationary, directly facing, and within $1-2 \mathrm{~cm}$ of the guillotine door. The door was then raised and the lamp left on for $5 \mathrm{sec}$. before being turned off for $10 \mathrm{sec}$. Three sec. after the lamp went off shock was applied to the grid. If $\mathrm{S}$ made no response after $7 \mathrm{sec}$. of shock the lamp came on and the shock went off simultaneously. An escape or avoidance response terminated the shock and turned on the lamp, after which the $S$ was left in the white side of the apparatus with the lamp on for $10 \mathrm{sec}$. Each $\mathrm{S}$ was given 20 trials a day for five days. There was an approximate $10 \mathrm{sec}$. interval between trials. Treatment of experimental and control Ss differed only in that one shock lead to the grid was disconnected when the control Ss were run. For both groups the frequencies of avoidances, escapes, and no responses were recorded as well as the response latency from the time the stimulus lamp went off. Avoidance responses made prior to the offset of

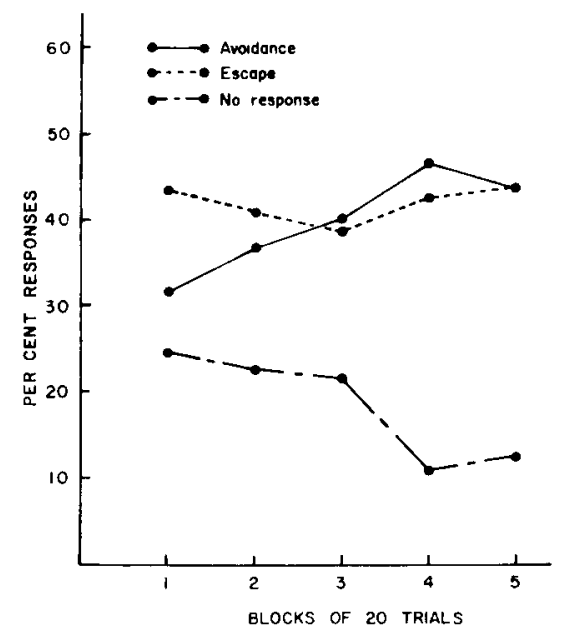

Fig. 1. Per cent avoidance, escape, and no response trials of the experimental Ss, for five daily blocks of 20 trials. 


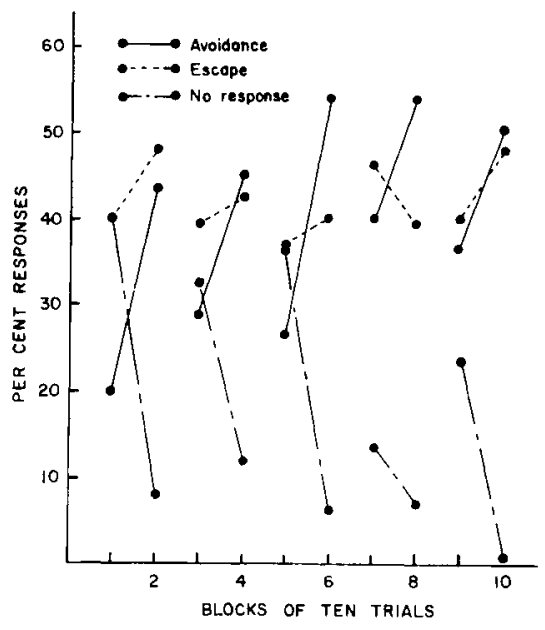

Fig. 2. Per cent avoidance, escape, and no response trials of the experimental Ss shown as the change between the first and last 10 trials each day.

the stimulus lamp were recorded as having a zero latency.

\section{Results and Discussion}

The percent of no response trials, escapes, and avoidances for the blocks of 20 daily trials are shown in Fig. 1. The percentage of escape trials is rather consistent across blocks of trials, and, except for the third block, never falls below $40 \%$. The avoidance responses rise from $32 \%$ to about $45 \%$ on the last two blocks of 20 trials. The percentage of no response trials gradually falls from $25 \%$ to about $11 \%$. Among the control Ss the daily percentages of no response for the five days were $95.6,96.9,99.4,99.4$, and 96.3 percent, respectively.

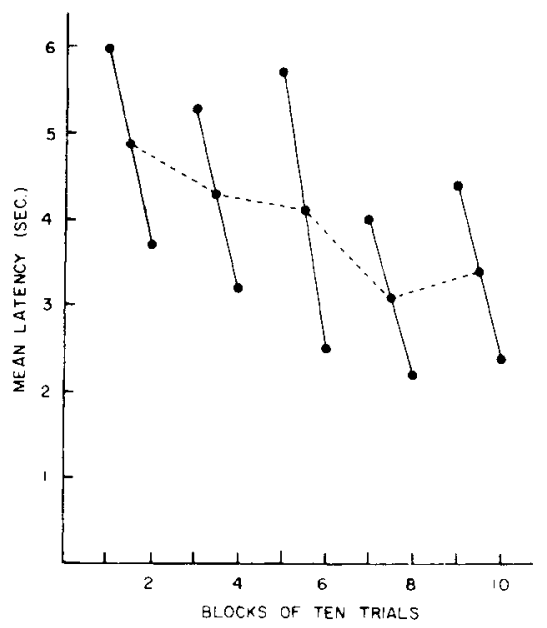

Fig. 3. Mean latency of response for the experimental Ss. The dashed line indicates the mean latencies of the five daily blocks of 20 trials. The change in mean latencies between the first and last 10 trials each day are shown by the solid lines.
During the experiment it became apparent that there was an increasing number of escape and avoidance responses occurring during the daily block of trials. The data was examined therefore in blocks of ten trials and is shown in Fig. 2. The curves show that the frequency of escape and avoidance responses was greater during the last 10 of each 20 daily trials with the single exception of the escape responses from trials 61-80. In addition, it may be seen that the terminal percentages of the last three blocks of 10 trials of avoidance responses were highly similar to each other. This may be an indication that the level of avoidance responding is approaching a maximum. The first 10 trials of each day produced an appreciably lesser number of avoidance responses than was found on the last 10 trials of the preceding day. A consistent and opposite effect may be seen in the no response trials.

Mean latencies of the control group were 9.7, 9.8, $10.0,10.0$, and $9.8 \mathrm{sec}$. for the five days, respectively. The mean latencies in seconds for the experimental Ss of the present study are shown in Fig. 3. It can be seen that the mean latencies of the daily trials, indicated by the dashed line, show a gradual decrease from an initial $4.8 \mathrm{sec}$. to 3.1 and $3.4 \mathrm{sec}$. on the last two blocks of 20 trials. The latencies of the first 10 trials were greater than the latencies of the second 10 trials on any given day. Furthermore, the initial 10 trials of each day had higher latencies than the last 10 trials of the preceding day. These results are consistent with those of the avoidance responses and the no response trials. It is possible to describe such results as demonstrating "forgetting" or as requiring "relearning" " on each of the daily trials. Although the latter explanation is perhaps the better of the two, it is more satisfactory to rely upon the descriptive data.

The positive findings of the present study possibly can be attributed to the one-way training, minimal shock, an advantageous number of trials, and the light conditions employed. The shock levels selected were determined by a series of experiments conducted prior to the present study. Experimental Ss typically did not show any overt behavioral response during early trials although they would pulsate with the shock on later trials. Preliminary studies indicated that fewer than 20 trials at a time were not effective but that many more than 20 seemed to cumulate the effects of the shock. These studies typically also employed a stimulus lamp that came on in the shock chamber prior to shock presentation, and which terminated with an avoidance or escape response. Such a procedure appears inadvisable since lizards and amphibians orient toward the light with a resultant restriction of behavior.

\section{References}

McGill, T. E. Response of the leopard frog to electric shock in an escape-learning situation. J. comp. physiol. Psychol., 1960, 53, 443-445.

Theios, J., \& Dunaway, I. E. One-way versus shuttle avoidance learning. Psychon. Sci., 1964, 1, 251-252. 